\title{
Influence of Intermolecular Interactions on the Structure of Copper Phthalocyanine Layers on Passivated Semiconductor Surfaces
}

\author{
Sanggyu Yim* and Tim S. Jones ${ }^{\dagger}$ \\ Department of Chemistry, Kookmin University, Seoul 136-702, Korea. *E-mail: sgyim@kookmin.ac.kr \\ ${ }^{\dagger}$ Department of Chemistry, University of Warwick, Coventry, CV4 7AL, UK \\ Received April 17, 2010, Accepted June 16, 2010
}

\begin{abstract}
The surface structures of copper phthalocyanine $(\mathrm{CuPc})$ thin films deposited on sulphur-passivated and plane perylene3,4,9,10-tetracarboxylic dianhydride (PTCDA)-covered InAs(100) surfaces have been studied by low energy electron diffraction (LEED) and van der Waals (vdW) intermolecular interaction energy calculations. The annealing to $300{ }^{\circ} \mathrm{C}$ and $450{ }^{\circ} \mathrm{C}$ of $\left(\mathrm{NH}_{4}\right)_{2} \mathrm{~S}_{\mathrm{x}}$-treated $\mathrm{InAs}(100)$ substrates produces a $(1 \times 1)$ and $(2 \times 1)$ S-passivated surface respectively. The $\mathrm{CuPc}$ deposition onto the PTCDA-covered InAs(100) surface leads to a ring-like diffraction pattern, indicating that the $2 \mathrm{D}$ ordered overlayer exists and the structure is dominantly determined by the intermolecular interactions rather than substrate-molecule interactions. However, no ordered LEED patterns were observed for the CuPc on S-passivated InAs(100) surface. The intermolecular interaction energy calculations have been carried out to rationalise this structural difference. In the case of CuPc unit cells on PTCDA layer, the planar layered $\mathrm{CuPc}$ structure is more stable than the $\alpha$-herringbone structure, consistent with the experimental LEED results. For CuPc unit cells on a S- $(1 \times 1)$ layer, however, the $\alpha$-herringbone structure is more stable than the planar layered structure, consistent with the absence of diffraction pattern. The results show that the lattice structure during the initial stages of thin film growth is influenced strongly by the intermolecular interactions at the interface.
\end{abstract}

Key Words: CuPc, Intermolecular interactions, Passivation, InAs, LEED

\section{Introduction}

There has been considerable interest in the growth of ordered thin films of macrocyclic molecules and their heterostructures for the application in a wide range of electronic and optoelectronic devices. ${ }^{1,2}$ The structural quality of the films depends strongly on the nature of the interface formed between layers of different materials. Perylene-3,4,9,10-tetracarboxylic dianhydride (PTCDA) and copper phthalocyanine $(\mathrm{CuPc})$, are two examples of the types of molecules commonly used in device applications such as light-emitting diodes and photovoltaics. ${ }^{1}$ From an energetic perspective, the surface overlayer structure of organic molecular thin films reflects the delicate balance of the intralayer energy associated with interactions between molecules in the overlayer $\left(E_{\text {intra }}\right)$ and the overlayer-substrate interface energy $\left(E_{\text {inter }}\right)$. It is more precise to describe this energetic balance in terms of the relative magnitudes of the corresponding elastic constants, $c_{\text {intra }}$ and $c_{\text {inter, }}$ which are determined from the second derivative, $\mathrm{d}^{2} E / \mathrm{d} x^{2}$, at the potential energy (PE) minima of $E_{\text {intra }}$ and $E_{\text {inter }}$ respectively, where $x$ is a coordinate within the overlayer or along the overlayer-substrate interface. ${ }^{3}$

Organic molecules are bonded to each other by relatively weak van der Waals (vdW) forces and form molecular crystals whose structure is determined by the optimisation of intermolecular interactions. The interfacial interaction with other organic crystals, or with relatively inert substrates, is also likely to be of the vdW variety, and highly crystalline structures can be formed without any requirement for lattice-matching between dissimilar materials at the interface. ${ }^{1,4}$ However, the creation of ordered molecular thin films on inorganic semiconductor surfaces is frequently difficult due to a large lattice mismatch between the organic film and the underlying substrate. Ordered growth on III-V compound semiconductor substrates is particularly problematic as the surface electronic structure is dominated by site-specific dangling bonds (dbs). ${ }^{5,6}$ This localised interaction and consequential limited lateral diffusion can hinder the formation of ordered crystalline domains when large organic molecules are deposited on the clean surface.

Ordered structures can be formed if the dbs are chemically passivated with some suitable species. Passivation of III-V semiconductor surfaces with sulphur atoms has been widely studied since it is well known that electrical properties are tremendously improved by passivation. ${ }^{7}$ Several methods of applying sulphur have been performed, including solution-based methods, ${ }^{7,8}$ gasbased methods ${ }^{9,10}$ and by the use of an electrochemical cell. ${ }^{11}$ Most studies have focused on the low index surfaces of $\mathrm{GaAs}^{8,12}$ and $\operatorname{InP},{ }^{13,14}$ whereas there has been relatively little work on the surfaces of InAs and InSb. The first sulphide treatment on the surface of GaAs was reported using $\mathrm{Na}_{2} \mathrm{~S} \cdot 9 \mathrm{H}_{2} \mathrm{O}$ solution. ${ }^{7}$ Although this treatment produces a very distinct improvement in the photoluminescence (PL) intensity, the treated surface is covered with a crusty film of sodium sulfide which prevents performing needed surface analyses. Also undesirable is the presence of sodium, which is a notorious cause of degradation in its device application. On the other hand, the treatment with ammonium sulphide, $\left(\mathrm{NH}_{4}\right)_{2} \mathrm{~S}_{\mathrm{x}}$, solution is more effective and reproducible. The effect of $\left(\mathrm{NH}_{4}\right)_{2} \mathrm{~S}_{\mathrm{x}}$ treatment has been observed as follows: ${ }^{15}$ 1) enhancement in PL intensity similar to that of $\mathrm{Na}_{2} \mathrm{~S}$ treatment; 2) complete removal of oxygen atoms on the surface, which was not attained by $\mathrm{Na}_{2} \mathrm{~S}$ treatment; 3) clear dependence of the Schottky barrier height on the work function of the contact metal.

Another method to reduce the influence of the substrate is the formation of molecular heterostructures; the deposition of 
organic molecules on previously grown molecular thin films such as PTCDA which preferentially forms planar layers on most substrates. Indeed, in a molecular thin film free-base phthalocyanine $\left(\mathrm{H}_{2} \mathrm{Pc}\right) / \mathrm{PTCDA}$ heterostructure, the XRD and AFM studies showed that an initial crystalline PTCDA layer has a significant influence on the structural and morphological properties of a second layer. ${ }^{16,17}$ In this system, the $\mathrm{H}_{2} \mathrm{Pc}$ molecules stack flat-lying layer by layer while they form $\alpha$-herringbone structures when deposited on non-interacting substrates such as glass. More recently, we have shown that this structural modification in the double layer heterostructure is due to the intermolecular interactions between the two layers at the heterointerface using vdW intermolecular interaction energy calculations. ${ }^{18}$

In this paper we present the overlayer structures of $\mathrm{CuPc}$ deposited on S-passivated and PTCDA film-covered InAs(100) surfaces using experimental low energy electron diffraction (LEED) studies and vdW intermolecular interaction energy calculations since the interfacial interactions of both systems are of the vdW variety. The calculations provide a quantitative explanation of the influence of intermolecular interactions on the structure of subsequent $\mathrm{CuPc}$ layer.

\section{Experimental Details}

A microchannel plate LEED facility (Omicron) was used for collection of the diffraction patterns with a primary beam current of $0.1 \mathrm{nA}$. This low beam current allows for real time experiments of deposition with negligible beam induced damage. Low energy electron beam energies were used because of the large unit cells of organic molecular overlayers.

Two types of InAs(100) substrates were used for preparing sulphur-passivated surfaces. One was used without any pretreatment (Atomergic Chemetal Corp., n-type, undoped) and the other was cleaned by ion bombardment and annealing (IBA) technique. IBA treatment was carried out in situ, after initial degassing at $250{ }^{\circ} \mathrm{C}$ for several hours, by two or three 15 minute cycles of simultaneous $\mathrm{Ar}^{+}$ion sputtering and annealing $\left(\mathrm{Ar}^{+}\right.$ energy of $500 \mathrm{eV}$ and substrate temperature of $300^{\circ} \mathrm{C}$ ), followed by post-bombardment annealing at $300{ }^{\circ} \mathrm{C}$ for 20 minutes. Grazing incidence ions were used for the sputtering to reduce structural damage. ${ }^{19}$ Further heating to $450-475^{\circ} \mathrm{C}$ was necessary to achieve the characteristic $(4 \times 2) / \mathrm{c}(8 \times 2)$ LEED pattern for the reconstructed (100) surface. Sulphur passivation was carried out ex situ with a wet chemical method. The untreated and IBA-treated InAs(100) samples were degreased by acetone and ethanol for 15 minutes each in a ultrasonic bath, and then rinsed by deionised water. The samples were dipped into an $\left(\mathrm{NH}_{4}\right)_{2} \mathrm{~S}_{\mathrm{x}}$ solution $\left(\mathrm{BDH}\right.$, minimum assay of $20 \%$ as $\left.\left(\mathrm{NH}_{4}\right)_{2} \mathrm{~S}\right)$ for 1 hour at room temperature, and then rinsed in a running deionised water. Finally they were dried in a flux of dry nitrogen. The samples were introduced into the ultrahigh vacuum (UHV) chamber with a base pressure of $<4 \times 10^{-10}$ mbar within 10 minutes after ex situ passivation process, and annealed at various temperatures up to $600{ }^{\circ} \mathrm{C}$ for 30 minutes by resistive heating; the annealing temperature measured using a W-5\%Re/ $\mathrm{W}-26 \%$ Re thermocouple mounted on the manipulator in close proximity to the sample.
PTCDA-covered InAs(100) substrate was prepared in situ. Commercially available PTCDA (Fluka, 98\%) powder was purified using temperature gradient vacuum sublimation. The purified PTCDA molecules were then outgassed in the UHV system for 20 hours before deposition, which involved sublimation from a miniature Knudsen effusion cell (K-cell). The IBAtreated InAs(100) substrate was held at room temperature.

$\mathrm{CuPc}$ deposition onto the S-passivated and the PTCDAcovered InAs(100) substrate was also carried out in situ. The process of the purification, introduction to the K-cell and sublimation of CuPc (Aldrich, 97\%) was similar to that of PTCDA molecules.

\section{Experimental Results}

Sulphur passivated substrate. The LEED patterns of sulphur passivated InAs(100) samples were observed to be consistent with those previously reported by Fukuda et al. ${ }^{20} \mathrm{~A}(1 \times 1)$ diffraction pattern was observed after annealing at $300{ }^{\circ} \mathrm{C}$ and it is reconstructed into the $(2 \times 1)$ structure upon annealing at 400 ${ }^{\circ} \mathrm{C}$. The slight increase of annealing temperature had no apparent effect on the quality of the diffraction patterns, although the sharpest pattern was obtained when the surface was annealed at $450{ }^{\circ} \mathrm{C}$. The same reconstructions occurred on the sulphur passivated surfaces of IBA-treated InAs(100) samples. Upon further annealing above $550{ }^{\circ} \mathrm{C}$ the $(2 \times 1)$ diffraction patterns disappeared remaining diffuse background for the untreated InAs(100) and reappearance of clean substrate diffraction patterns for the IBA-treated InAs(100) sample. This indicates that surface sulphur atoms have already been desorbed.

PTCDA-covered substrate. A LEED pattern of the clean In As(100)- $(4 \times 2) / c(8 \times 2)$ surface recorded with a beam energy of $14 \mathrm{eV}$ is shown in Fig. 1(a). Upon PTCDA deposition at room temperature the substrate spots gradually lose intensity and faint overlayer spots appear superimposed on the substrate pattern. Figure 1(b) shows the LEED pattern obtained at $14 \mathrm{eV}$ after deposition of 1 - 2 ML of PTCDA representing the superposition of some substrate spots and the ring-like feature of the overlayer. This ring-like feature is similar to that observed in previous LEED studies. ${ }^{21}$ They also investigated the overlayer structures using STM and suggested that the observed diffraction pattern is generated by the superposition of two domains, each comprising a slightly distorted rectangular unit cell in which two PTCDA molecules form a usual 'herringbone' type structure.

From comparison of the overlayer diffraction spots with the position of the substrate spots in Fig. 1(b), an estimation of the overlayer unit cell dimensions was made. The lengths of the unit cell vectors are $(19.9 \AA \pm 2.0 \AA) \times(12.5 \AA \pm 2.0 \AA)$ and an included angle of $88^{\circ} \pm 2^{\circ}$ with the PTCDA unit cell rotational angle with respect to the InAs(100) substrate unit vector of $46^{\circ}$, which are good agreement with the values previously found by STM study $^{21}$ and those for the (102) plane of the bulk crystal. ${ }^{22}$ Therefore, it is reasonable to be assumed that the PTCDA molecules adopt the standard herringbone-type arrangement. The schematic of the reciprocal space structure is shown in Fig. 1(d). The circles indicate the PTCDA diffraction spots and the squares indicate the substrate spots. The filled shapes represent the 

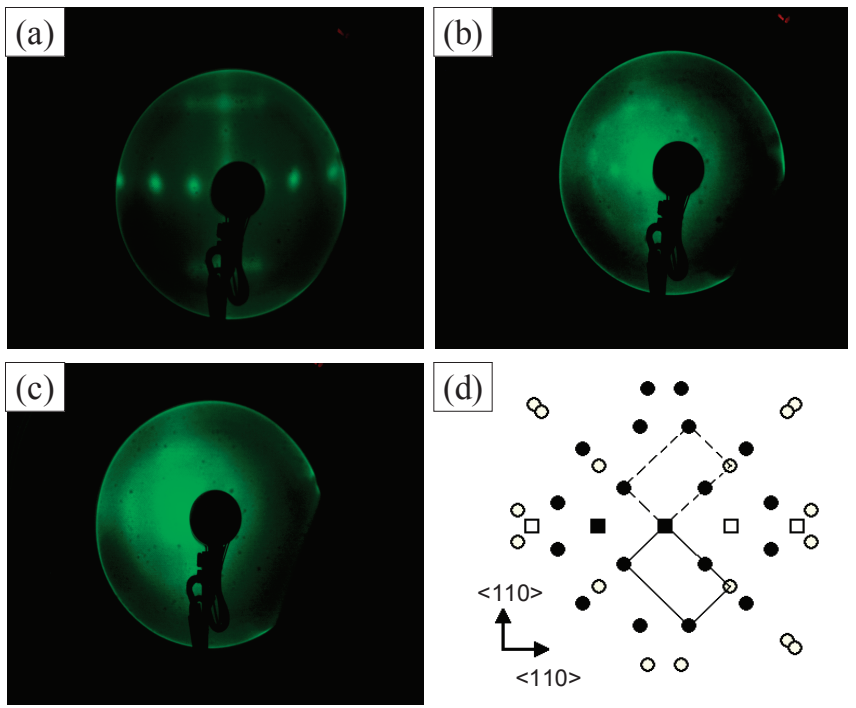

Figure 1. LEED patterns of (a) clean $\operatorname{InAs}(100)-(4 \times 2) / \mathrm{c}(8 \times 2)$, (b) $1 \sim 2$ ML PTCDA and (c) $3 \sim 4$ ML PTCDA deposited on (a). The electron beam energies were $14 \mathrm{eV}$. A schematic of the reciprocal space structure is shown in (d). The squares represent the substrate spots, and the circles indicate the overlayer reciprocal structures with the filled shapes indicating the spots observed in the LEED pattern shown in (b). The two overlayer reciprocal lattice unit cells are indicated by the dashed and solid lines.

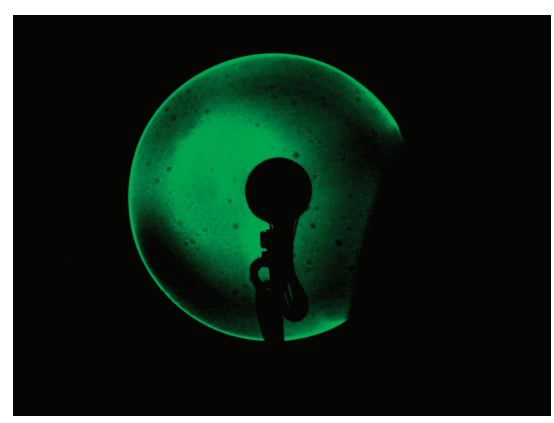

Figure 2. A LEED pattern of $\sim 2 \mathrm{ML} \mathrm{CuPc/PTCDA} / \operatorname{InAs}(100)$. The electron beam energy was $14 \mathrm{eV}$.

spots observed in the actual LEED pattern shown in Fig. 1(b). The two domains of reciprocal lattice unit cells are indicated by the dashed and solid lines. The LEED pattern associated with the overlayer becomes more diffuse with increasing coverage and the substrate spots rapidly disappeared. The LEED pattern in Fig. 1(c) corresponds to 3 - 4 ML PTCDA deposition.

CuPc growth. During the initial stage of CuPc deposition onto the 3 - 4 ML PTCDA-covered InAs(100) substrate, the ring-like diffraction pattern associated with the underlying PTCDA layer is augmented by new ring-like feature with slightly smaller radius. A LEED pattern is shown in Fig. 2 after $\sim 2$ ML CuPc deposition, recorded with electron beam energy of $14 \mathrm{eV}$. An existence of the diffraction pattern indicates that 2D ordered $\mathrm{CuPc}$ overlayer forms on the PTCDA-covered InAs (100) surface, although the patterns are not that clearly defined. This fuzziness of diffraction pattern implies the lack of long range $2 \mathrm{D}$ order of $\mathrm{CuPc}$ overlayer, which may be due to the surface roughness, the lack of ideally large flat terraces, of under- lying PTCDA layer. Nevertheless, the ring-like diffraction features indicate that the overlayer $\mathrm{CuPc}$ unit cell does not have a fixed orientation with respect to the underlying PTCDA unit cell. This means that, in this system, the overlayer structure is dominantly determined by the intermolecular interactions, whereas the substrate-molecule (PTCDA-CuPc) interaction is not strong enough to govern the commensurate growth or rotational epitaxy.

The same experiments were carried out with $\mathrm{CuPc}$ deposited on S-passivated InAs(100), however no ordered LEED patterns were observed at any coverage. An annealing of $\mathrm{CuPc}$ deposited surface at various temperatures had no effect.

\section{Theoretical Details}

Intermolecular interaction energy calculations in this work were performed using a Lennard-Jones 9 - $6 \mathrm{vdW}$ interaction energy for all possible, non-bonded atom pairs, i.e.,

$$
E_{\mathrm{vdW}(\mathrm{LJ}-9-6)}=\sum \varepsilon_{i j}\left\{2\left(R_{i j}{ }^{*} / R_{i j}\right)^{9}-3\left(R_{i j}{ }^{*} / R_{i j}\right)^{6}\right\}
$$

where $R_{i j}$ is the distance between the $i$ th and $j$ th atoms, $R_{i j}{ }^{*}$ is the minimum energy separation between atom $i$ and atom $j$, and $-\varepsilon_{i j}$ is the energy for the $i, j$ interaction attained at $R_{i j}=R_{i j}{ }^{*}$. Previously reported molecular mechanics (MM) force field parameters and structural parameter values (bond lengths and angles) ${ }^{23-25}$ were used for the calculations. Based on these molecular structures, we have calculated the intermolecular interaction energies of six different CuPc unit cell models; (i) the bulk $\alpha$-herringbone structure, (ii) a planar layered bulk structure, (iii) the $\alpha$-herringbone structure on PTCDA layer, (iv) a planar layered structure on PTCDA layer, (v) the $\alpha$-herringbone structure on S- $(1 \times 1)$ layer and (vi) a planar layered structure on $\mathrm{S}-(1 \times 1)$ layer.

Bulk CuPc unit cells. The intermolecular interaction energy of the $\alpha$-herringbone $\mathrm{CuPc}$ unit cell was calculated by varying tilt angles of the molecular plane, $\tau_{l}$ and $\tau_{2}$, and molecular orientation angles in the molecular plane, $\delta_{1}$, and $\delta_{2}$. The definitions of these angles were well described elsewhere. ${ }^{18}$ The $\alpha$-herringbone $\mathrm{CuPc}$ unit cell has a minimum energy of $-3.086 \mathrm{eV}$ at $\tau_{l}=$ $\tau_{2}=9.0^{\circ}, \delta_{1}=58.3^{\circ}$ and $\delta_{2}=31.7^{\circ}$. The vdW interaction energy surfaces for the $\alpha$-herringbone unit cell of CuPc are shown in Fig. 3: (a) shows the energy surface drawn with respect to the molecular plane tilt angles, $\tau_{1}$ and $\tau_{2}$, when the in-plane molecular rotational angles, $\delta_{l}$ and $\delta_{2}$, are fixed at $58.3^{\circ}$ and $31.7^{\circ}$ respectively, and (b) shows the energy surface drawn with respect to $\delta_{1}$ and $\delta_{2}$ with $\tau_{1}=\tau_{2}=9.0^{\circ}$. The intermolecular interaction energy of the CuPc molecules in the $\alpha$-herringbone unit cell can be divided into two types; (1) the interaction energy between molecules with the same height $\left(\mathrm{E}_{\leftrightarrow}\right)$, and (2) the interaction energy with molecules positioned on the upper or lower layers $\left(\mathrm{E}_{\uparrow}\right)$. For the $\alpha$-herringbone unit cell, $\mathrm{E}_{\leftrightarrow}$ and $\mathrm{E}_{\uparrow}$ were calculated to be $-2.590 \mathrm{eV}$ and $-0.495 \mathrm{eV}$, respectively, at the total energy minimum.

The intermolecular interaction energy of the planar layered $\mathrm{CuPc}$ unit cell was calculated by varying parameters, $x, y$ and $z$. The lateral displacement parameters $(x, y)$ of a molecule in the upper 2D quadrate with respect to the corresponding mole- 
(a)

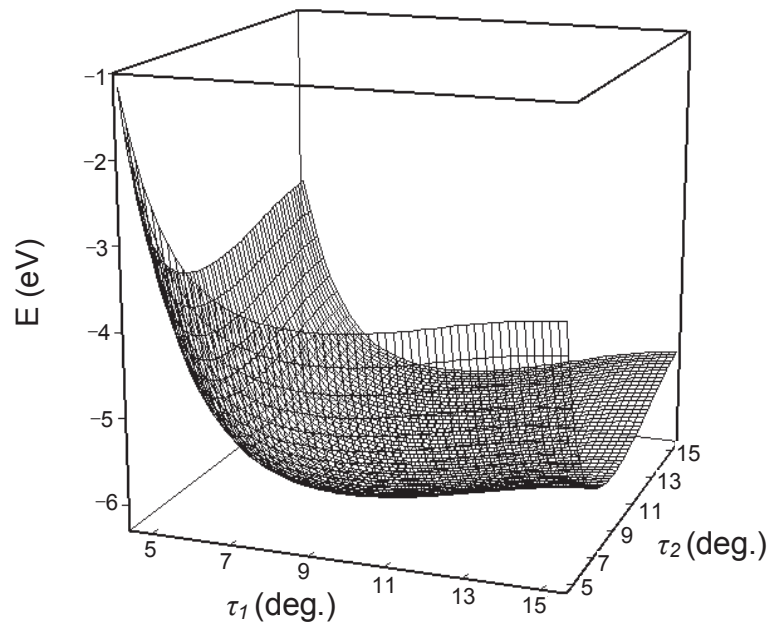

(b)

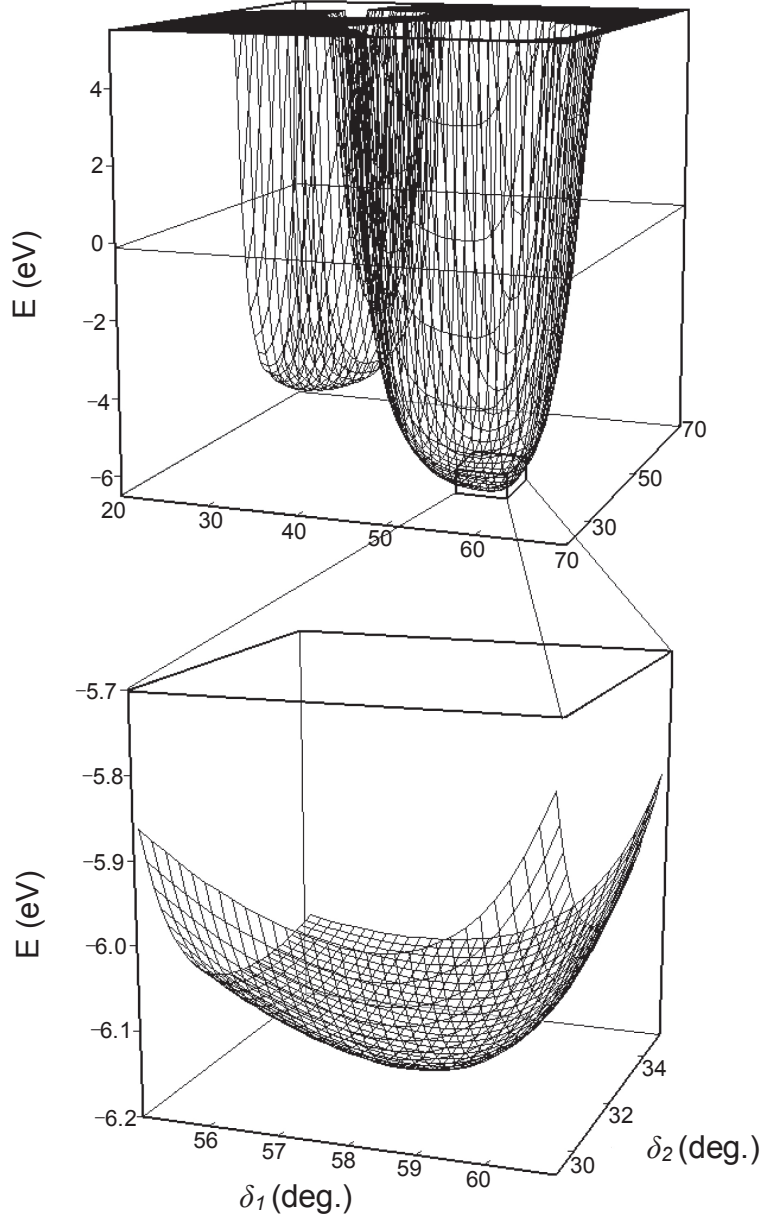

Figure 3. Intermolecular interaction energy surface diagrams for the bulk $\alpha$-herringbone $\mathrm{CuPc}$ unit cell as a function of (a) $\tau_{1}$ and $\tau_{2}$ at $\delta_{1}=$ $58.3^{\circ}$ and $\delta_{2}=31.7^{\circ}$, and (b) $\delta_{1}$ and $\delta_{2}$ at $\tau_{1}=\tau_{2}=9.0^{\circ}$. The lower energy surface is an expansion around the energy minimum.

cule in the lower quadrate were varied from $-7 \AA$ to $7 \AA$ with an increment of $0.1 \AA$. The interplanar stacking distance between two layers, $z$, was varied from $0 \AA$ to $9 \AA$ with an increment of $0.01 \AA$. We have recently reported that a $2 \mathrm{D}$ quadratic $\mathrm{CuPc}$ unit cell has its minimum intermolecular interaction energy at the lattice parameters, $a$ and $b$, of $13.85 \AA$, and a molecular rota- (a)

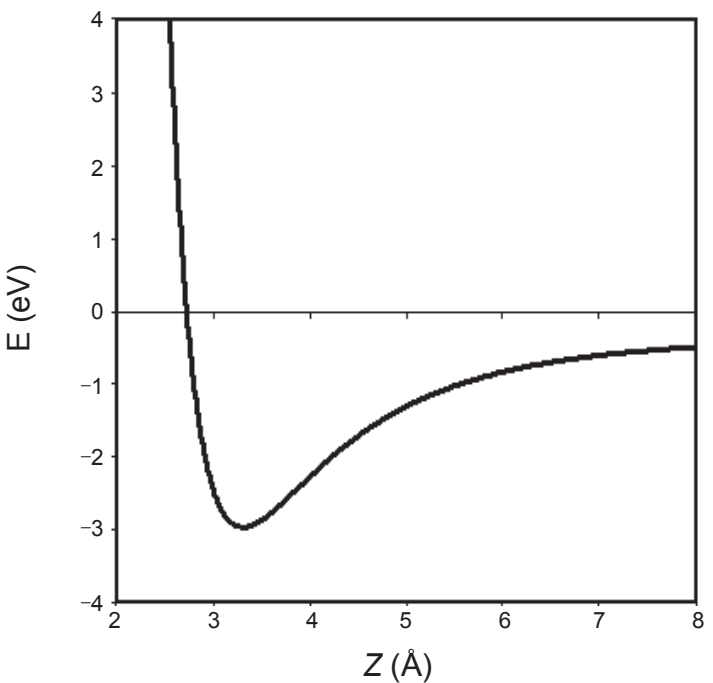

(b)

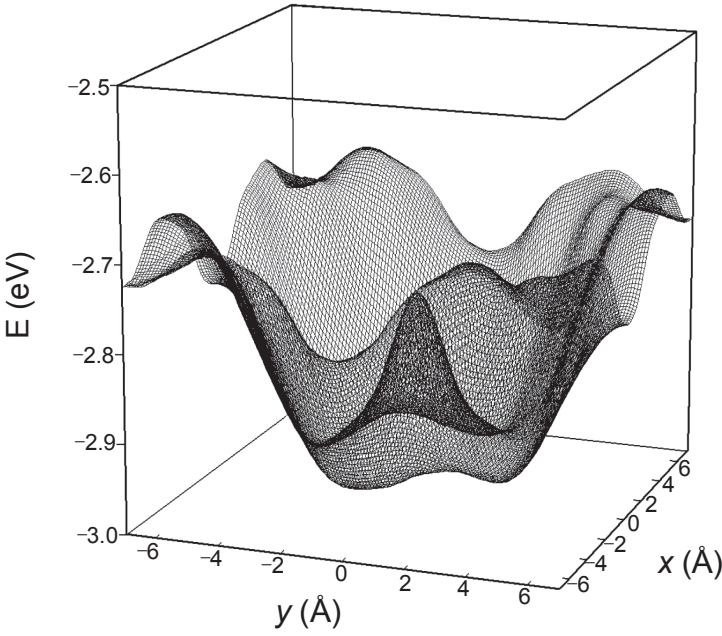

Figure 4. Intermolecular interaction energies for the bulk planar layered CuPc unit cell: (a) energy curve plotted as a function of interplanar stacking distance, $z$, at $x=3.2 \AA$ and $y=0.9 \AA$, and (b) energy surface drawn as a function of the parallel displacements, $x$ and $y$, at $z=3.31 \AA$.

tional angle in a plane, $\delta$, of $27.3^{0} .{ }^{26,27}$ These fixed 2D quadrate parameter values were used for the calculation. The planar layered $\mathrm{CuPc}$ unit cell has degenerate energy minima of -2.963 $\mathrm{eV}$ at $x= \pm 3.2 \AA, y= \pm 0.9 \AA$ and $z=3.31 \AA$, which is slightly greater than that of the $\alpha$-herringbone unit cell, $-3.086 \mathrm{eV}$. The $\mathrm{vdW}$ interaction energy curves for the planar layered unit cell of CuPc are shown in Fig. 4. Figure 4(a) shows the energy curve plotted with respect to the interplanar distance between two 2D quadrates, $z$. The parallel displacement parameters, $x$ and $y$, are fixed at $3.2 \AA$ and $0.9 \AA$ respectively. Figure 4(b) shows the energy surface drawn with respect to $x$ and $y$ with $z$ fixed at $3.31 \AA$. At the energy minimum for the molecule in a planar layered $\mathrm{CuPc}$ unit cell, the interaction energy between the molecules within the layer, $\mathrm{E}_{\leftrightarrow}$, is $-0.388 \mathrm{eV}$ and the interaction energy with molecules in different layers, $\mathrm{E}_{\mathfrak{\downarrow}}$, is $-2.575 \mathrm{eV}$.

CuPc unit cells on a PTCDA layer. Figure 5 shows schematic models for the $\alpha$-herringbone (Fig. 5a) and planar layered (Fig. 5 b) $\mathrm{CuPc}$ unit cells on a PTCDA layer. For the calculations, the lattice parameters of the underlying 2D PTCDA layer consisting 
(a)

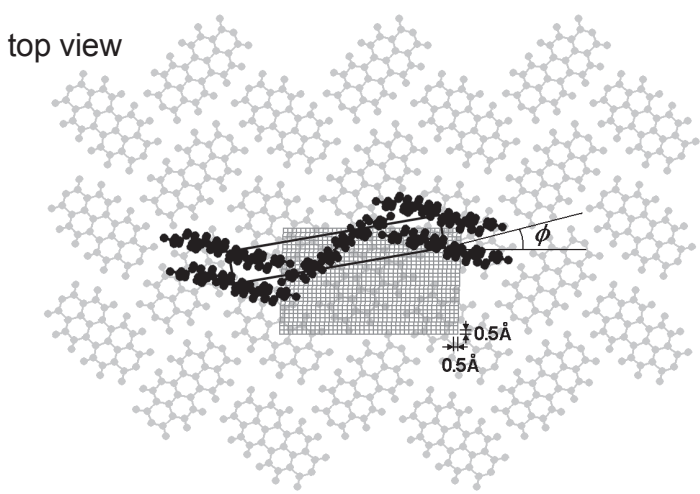

side view

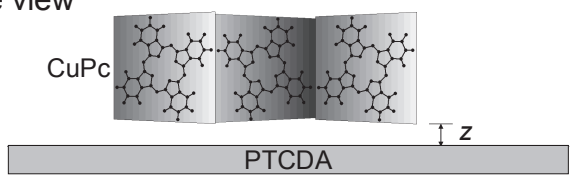

(b)

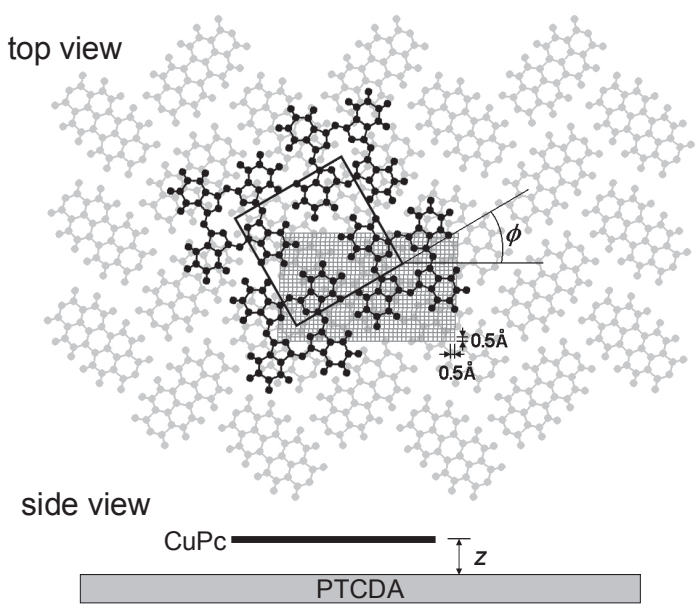

Figure 5. Schematic models for (a) the $\alpha$-herringbone $\mathrm{CuPc}$ unit cell on a PTCDA layer, and (b) the planar layered $\mathrm{CuPc}$ unit cell on a PTCDA layer. $z$ represents the stacking distance between the CuPc unit cell and the PTCDA layer. The black rectangle in (a) and square in (b) represent the $\mathrm{CuPc}$ unit cells. The PTCDA unit cells (grey quadrangle) are divided into 960 square meshes with a length and width of $0.5 \AA$. ø represents the azimuthal rotational angle of $\mathrm{CuPc}$ unit cell with respect to the PTCDA unit vector.

of 26 molecules were obtained in section 3.2. The intermolecular interaction energies between the CuPc unit cell and the underlying PTCDA layer, $\mathrm{E}_{\mathfrak{\downarrow}}$, were calculated with respect to the stacking distance between the two layers, $z$. At each point of $z$, the energy was determined by assuming that the $\mathrm{CuPc}$ unit cell can be placed arbitrarily on the PTCDA layer since the exact position of the CuPc unit cell is not determined by LEED study. This assumption is generally valid since organic molecules such as $\mathrm{CuPc}$ and PTCDA are bonded to each other by relatively weak $\mathrm{vdW}$ forces and their lattice parameter values are quite large, consequently the interfacial interaction between the two different materials does not require any lattice matching. The interfacial interaction energy of a CuPc/PTCDA heterostructure depends primarily on the stacking distance between the two layers, with relatively little influence from the lateral displace-

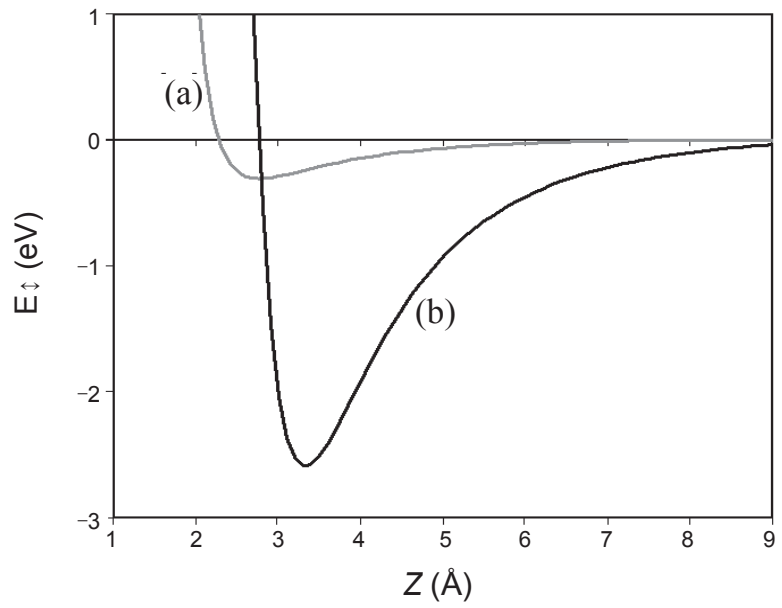

Figure 6. Intermolecular interaction energy curves between the PTCDA layer and (a) the $\alpha$-herringbone CuPc unit cell and (b) the planar layered $\mathrm{CuPc}$ unit cell as a function of the stacking distance, $z$.

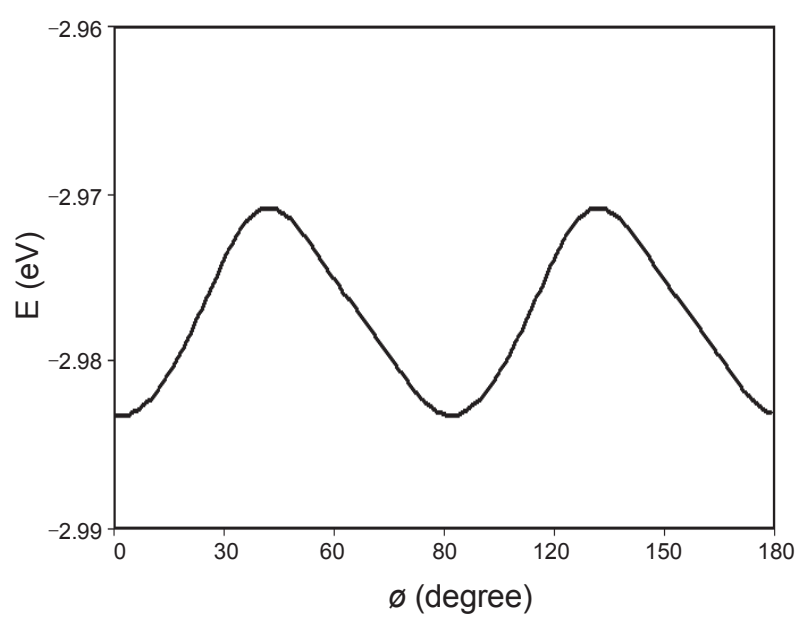

Figure 7. The energy curve as a function of the azimuthal rotational angle of $\mathrm{CuPc}$ unit cell with respect to the PTCDA unit vector, $\varnothing$, at $z=3.32 \AA$.

ment of CuPc unit cell. To calculate the intermolecular interaction energy between the arbitrarily positioned $\mathrm{CuPc}$ unit cell and the PTCDA layer, the underlying PTCDA unit cell (grey quadrangle in the figure) was divided into $960(40 \times 24)$ subunit meshes with a length and width of $0.5 \AA$. The rotational angle of the $\mathrm{CuPc}$ unit cell (black quadrangle in the figure) with respect to the PTCDA unit cell vector, $\varnothing$, was varied from $0^{\circ}-180^{\circ}$ with an increment of $1^{\circ}$. At a certain stacking distance, $z$, and $\mathrm{CuPc}$ unit cell rotational angle, $\varnothing$, the interaction energies were then calculated when the centre of the $\mathrm{CuPc}$ unit cell was positioned on each mesh point, and average values were taken. Then the energy value at a certain $z$ was finally determined by averaging the energy values taken at each ø. Once the stacking distance, $z$, was determined, any fluctuation of the interaction energy due to a change in lateral displacement or the rotation of the $\mathrm{CuPc}$ unit cell was found to be insignificant.

Figure 6 shows the energy curves between the two layers, $E_{\uparrow}$, with respect to the stacking distance, $z$. In the case of the $\alpha$-herr- 
(a)

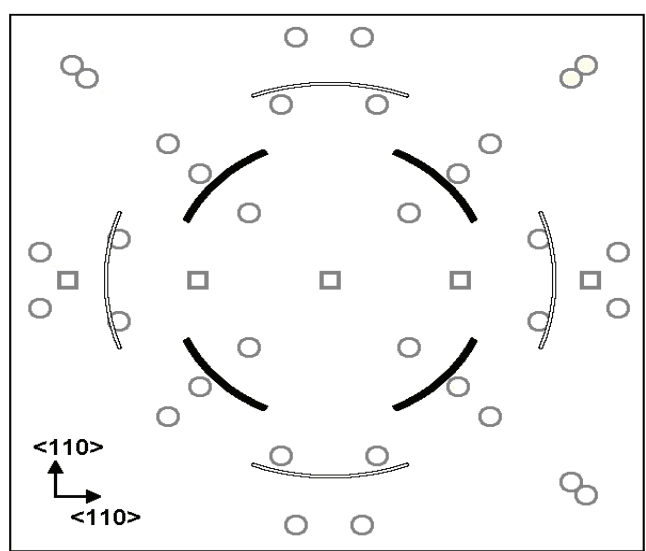

(b)

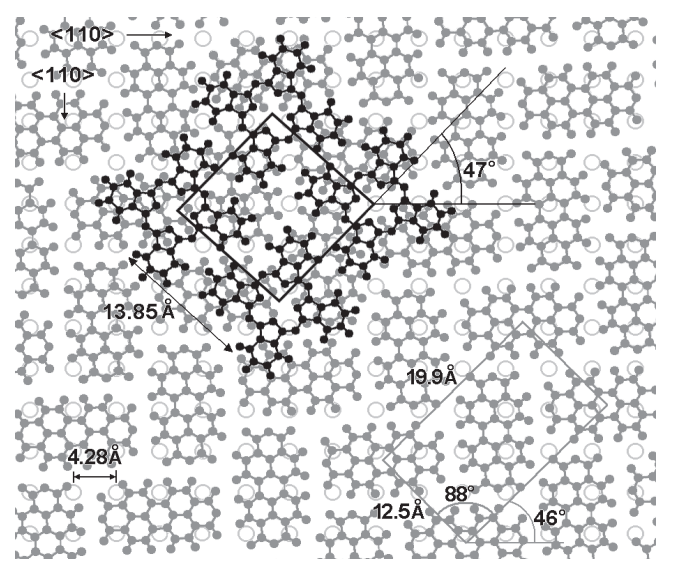

Figure 8. A schematic of (a) the reciprocal space structure and (b) the real space structure proposed for $\mathrm{CuPc} / \mathrm{PTCDA} / \operatorname{InAs}(100)$. In (a), the black arcs represent the quadratic $\mathrm{CuPc}$ structures with $\varnothing=1^{\circ} \pm 20^{\circ}$. The small gray circles and rectangles indicate the PTCDA and InAs(100) reciprocal structures respectively. In (b), the black square and grey quadrangle represent the surface unit cell of the $\mathrm{CuPc}$ and underlying PTCDA respectively. The structures are drawn with respect to the ideal terminated $\operatorname{InAs}(100)$ surface (grey empty circles). The exact sites over which the $\mathrm{CuPc}$ and PTCDA are positioned are not known.

ingbone structure (Fig. 6a), the energy minimum is $-0.307 \mathrm{eV}$ at $z=2.76 \AA$, whilst for the planar layered structure (Fig. 6b), it is $-2.589 \mathrm{eV}$ at $z=3.32 \AA$. For a given $z$, any lateral displacement and the rotation of a $\mathrm{CuPc}$ unit cell has a minor effect on the energy variation. For example, in the case of the $\alpha$-herringbone CuPc unit cell on PTCDA at $z=2.76 \AA$, the variation in $\mathrm{E}_{\uparrow}$ is $0.098 \mathrm{eV}$, from $-0.356 \mathrm{eV}$ to $-0.258 \mathrm{eV}$, and for the planar layered unit cell at $z=3.32 \AA$, it is $0.125 \mathrm{eV}$, from $-2.624 \mathrm{eV}$ to $-2.499 \mathrm{eV}$. This small variation is also consistent with our assumption that the interaction energy between the $\mathrm{CuPc}$ unit cell and the PTCDA layer at a specific $z$ can be regarded as an average value irrespective of their exact position. Therefore, the total energy, $\mathrm{E}\left(=\mathrm{E}_{\uparrow}+\mathrm{E}_{\leftrightarrow}\right)$, of the planar layered $\mathrm{CuPc}$ unit cell on a PTCDA layer is $-2.977 \mathrm{eV}$, which is lower than that of a $\alpha$-herringbone $\mathrm{CuPc}$ unit cell on a PTCDA layer, $-2.897 \mathrm{eV}$. This is consistent with the existence of the 2D diffraction pattern observed in the experimental LEED results for the $\mathrm{CuPc}$ on a PTCDA-covered InAs(100) substrate.

One additional interesting fact is that, in the ring-like diffraction pattern (Fig. 2), some arcs are brighter. The brighter arcs (a)

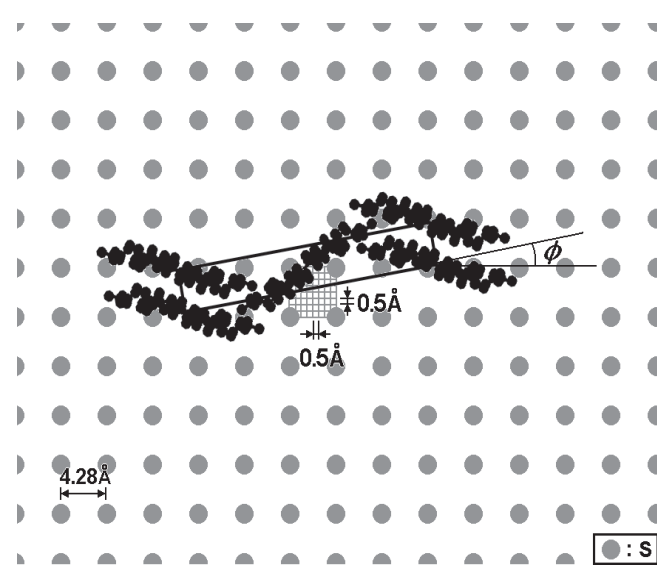

(b)

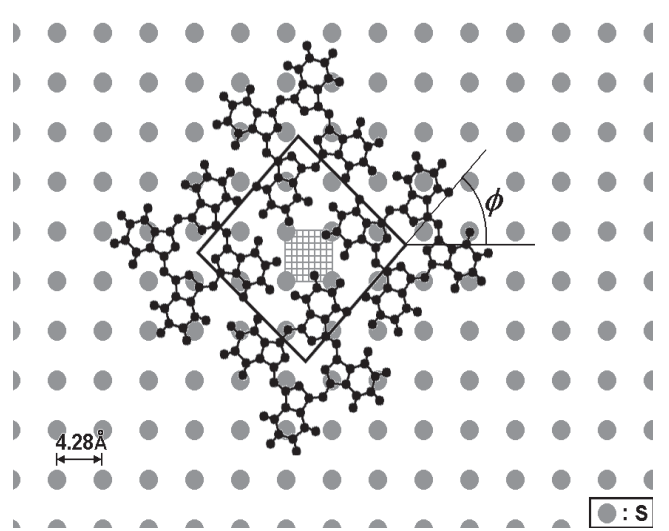

Figure 9. Top view schematic models for (a) the $\alpha$-herringbone $\mathrm{CuPc}$ unit cell on a S-(1×1) layer, and (b) the planar layered CuPc unit cell on a S- $(1 \times 1)$ layer. The black rectangle in (a) and square in (b) represent the $\mathrm{CuPc}$ unit cells. The $\mathrm{S}-(1 \times 1)$ unit cells (grey square) are divided into 81 square meshes with a length and width of $0.5 \AA$. ø represents the azimuthal rotational angle of $\mathrm{CuPc}$ unit cell with respect to the S- $(1 \times 1)$ unit vector.

are positioned diagonally, $\sim 45^{\circ}$ with respect to the reciprocal unit cell vectors of the InAs(100) substrate. This variation of brightness may be the result of the variation of the interaction energy with respect to the rotational angle of the $\mathrm{CuPc}$ unit cell, $\varnothing$. The energy curve as a function of $\varnothing$ at $z=3.32 \AA$ is shown in Fig. 7. The energies are fluctuated with a $90^{\circ}$ periodicity and has a minimum of $-2.983 \mathrm{eV}$ at $\varnothing=1^{\circ}$ and a maximum of $-2.971 \mathrm{eV}$ at $\varnothing=42^{\circ}$, although the variation is not that great. The expected reciprocal LEED pattern of quadratic CuPc unit cells with lattice dimensions of $13.85 \AA \times 13.85 \AA$ and the rotational angle with respect to the PTCDA unit cell vector, $\varnothing$, of $1^{\circ} \pm 20^{\circ}$ are drawn in Fig. 8(a), which is consistent with the LEED pattern observed in Fig. 1(b) and Fig. 2. The black arcs are drawn for quadratic $\mathrm{CuPc}$ unit cells and the grey squares represent the substrate spots, with grey circles indicating PTCDA reciprocal structures. Real space structures expected from the diffraction patterns and intermolecular interaction energy calculations are shown schematically in Fig. 8(b). The black square and grey quadrangle represent the surface unit cell of CuPc and underlying PTCDA respectively. The exact lattice sites over which the CuPc and 


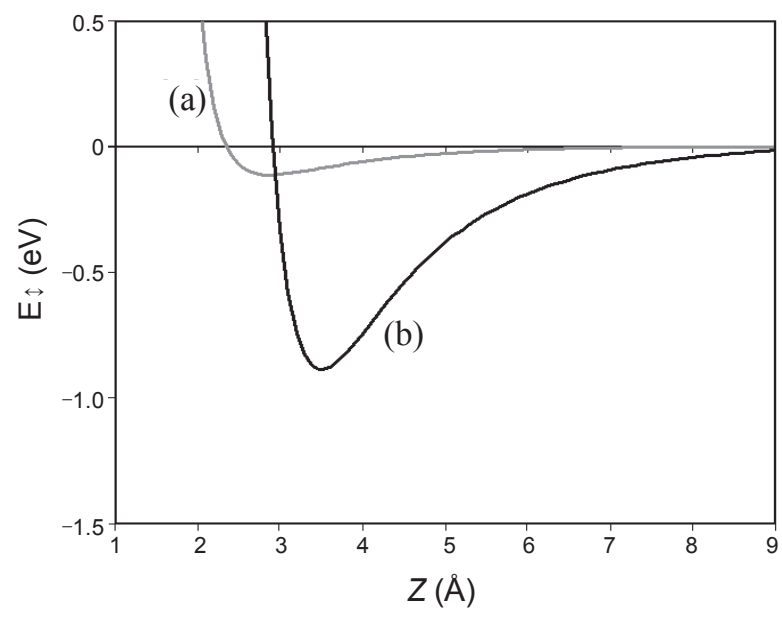

Figure 10. Intermolecular interaction energy curves between the S$(1 \times 1)$ layer and (a) the $\alpha$-herringbone CuPc unit cell and (b) the planar layered $\mathrm{CuPc}$ unit cell as a function of the stacking distance, $z$.

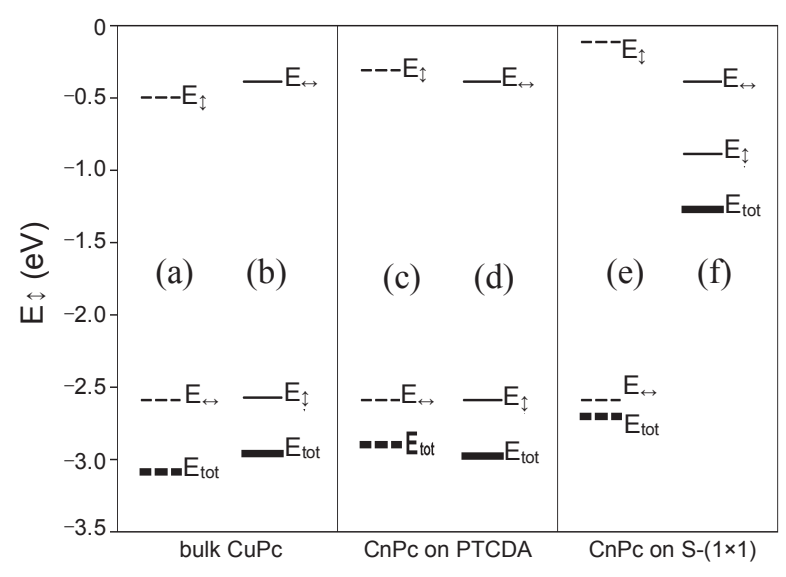

Figure 11. Energy level diagrams for six different $\mathrm{CuPc}$ unit cell models; (a) the bulk $\alpha$-herringbone structure, (b) the bulk planar layered structure, (c) the $\alpha$-herringbone structure on PTCDA, (d) the planar layered structure on PTCDA, (e) the $\alpha$-herringbone structure on S- $(1 \times 1)$ and (f) the planar layered structure on $\mathrm{S}-(1 \times 1)$.

PTCDA molecules are positioned are not known.

CuPc unit cells on a S-(1×1) layer. Figure 9 shows schematic models for the $\alpha$-herringbone (Fig. 9a) and planar layered (Fig. $9 b) \mathrm{CuPc}$ unit cells on a S- $(1 \times 1)$ layer. The intermolecular interaction energies between the $\mathrm{CuPc}$ unit cell and the underlying $\mathrm{S}$ - $(1 \times 1)$ layer, $\mathrm{E}_{\uparrow}$, were calculated using the same procedure described in the previous section. For the calculation, the underlying 2D S-(1×1) layer consisting of $441(21 \times 21)$ sulphur atoms were used. The underlying sulphur unit cell (grey square in the figure) was divided into $81(9 \times 9)$ square meshes with a length and width of $0.5 \AA$. The rotational angle of the $\mathrm{CuPc}$ unit cell (black quadrangle in the figure) with respect to the underlying $\mathrm{S}-(1 \times 1)$ unit cell vector, $\varnothing$, was varied from $0^{\circ}-180^{\circ}$ with an increment of $1^{\circ}$. At a certain stacking distance, $z$, and $\mathrm{CuPc}$ unit cell rotational angle, $\varnothing$, the interaction energies were calculated when the centre of the $\mathrm{CuPc}$ unit cell was positioned on each mesh point, and averaged. Finally, the energy value at a certain $z$ was determined by averaging the energy values taken at each $\varnothing$.

Figure 10 shows the interlayer energy curves with respect to the stacking distance $z$. In the case of the $\alpha$-herringbone structure (Fig. 10a), the energy minimum is $-0.113 \mathrm{eV}$ at $z=2.87 \AA$, while for the planar layered structure (Fig. 10b), it is $-0.886 \mathrm{eV}$ at $z=$ $3.50 \AA$. For the $\alpha$-herringbone $\mathrm{CuPc}$ unit cell, the variation in $\mathrm{E}_{\uparrow}$ due to the lateral displacement and rotation of the $\mathrm{CuPc}$ unit cell at $z=2.87 \AA$ is $0.077 \mathrm{eV}$ and for the planar layered unit cell at $z=3.50 \AA$, it is $0.034 \mathrm{eV}$. The total energy, $\mathrm{E}\left(=\mathrm{E}_{\mathfrak{1}}+\mathrm{E}_{\leftrightarrow}\right)$, of $\alpha$-herringbone $\mathrm{CuPc}$ unit cell on the $\mathrm{S}-(1 \times 1)$ layer is -2.703 $\mathrm{eV}$, which is lower than that of a planar $\mathrm{CuPc}$ unit cell on a PTCDA layer, $-1.274 \mathrm{eV}$. These calculations imply that the $\mathrm{CuPc}$ molecules are deposited onto a S-passivated surface with a bulk-like herringbone structures instead of forming a planar 2D layer.

Three orientations of $\alpha$-herringbone crystallites have been reported when the $\mathrm{CuPc}$ molecules are deposited onto the noninteracting or weakly interacting substrates, which mainly depends on the nature of the substrates. They are parallel b-axis, perpendicular b-axis and standing b-axis orientations. ${ }^{28}$ At this stage, however, it is impossible to determine which orientation is the most favourable since we have calculated only for the parallel $b$-axis configuration. Nevertheless, the calculations indicate that the CuPc molecules cannot form flat-lying 2D ordered structure on a S-(1×1) layer, since the substrate molecule $(\mathrm{S}-\mathrm{CuPc})$ interactions in this system cannot overcome the penalty of the intermolecular interaction energy arising from the deviation of their native $\alpha$-herringbone structure. The absence of the ordered LEED pattern in this system may be rationalised by this energetic disadvantage of the planar layered 2D structure.

The energy levels for the six $\mathrm{CuPc}$ unit cell structures are shown in Fig. 11. The two in the left box, (a) and (b), represent the energy levels for bulk $\mathrm{CuPc}$ unit cells and the two in the middle box, (c) and (d), for the CuPc unit cells on a PTCDA layer, and the two in the right box, (e) and (f), correspond to the $\mathrm{CuPc}$ unit cells on a S- $(1 \times 1)$ layer. The energy levels for the $\alpha$-herringbone unit cells are displayed with dashed lines and those for planar layered unit cells with solid lines.

In the case of the bulk unit cells, the intermolecular interaction energy within the layer, $E_{\leftrightarrow}$, for the $\alpha$-herringbone structure is lower than that of the interaction energy with molecules in different layers, $E_{\downarrow}$, for the planar layered structure. The level of $\mathrm{E}_{\downarrow}$ for the $\alpha$-herringbone structure is also lower than that of $\mathrm{E}_{\leftrightarrow}$ for the planar layered structure. Consequently, the $\alpha$-herringbone structure is more stable than the planar layered structure by $\sim 3 \%$, consistent with the existence of the $\alpha$-herringbone $\mathrm{CuPc}$ structure rather than a planar layered structure when $\mathrm{CuPc}$ molecules are deposited on non-interacting substrates. For the $\mathrm{CuPc}$ unit cells on PTCDA, however, the total energy levels are reversed since the $E_{\downarrow}$ level for the $\alpha$-herringbone unit cell on the PTCDA layer is higher than that in the corresponding bulk structure. During the initial stage of $\mathrm{CuPc}$ deposition on a planar PTCDA layer, this minimum energy difference seems to determine the structure of the $\mathrm{CuPc}$ films. For the monolayer deposition of CuPc on PTCDA, the total energy of the planar layered film is $-287.2 \mathrm{~kJ} / \mathrm{mol}$, which is $\sim 3 \%$ more stable than that of the $\alpha$-herringbone structure $(-279.5 \mathrm{~kJ} / \mathrm{mol})$. For the 
CuPc unit cells on S- $(1 \times 1)$ layer, however, the interlayer interaction energy, $\mathrm{E}_{\downarrow}$ of planar layered $\mathrm{CuPc}$ unit cell is much higher than that on the PTCDA layer. Consequently, the total energy of the $\alpha$-herringbone monolayer film is much more stable than that of the planar layered structure. These calculation results provide a quantitative explanation for the existence and absence of the diffraction pattern for the CuPc molecules on a PTCDA layer and sulphur layer respectively.

\section{Conclusion}

The $\left(\mathrm{NH}_{4}\right)_{2} \mathrm{~S}_{\mathrm{x}}$ treatment on InAs(100) substrate and subsequent annealing to $300{ }^{\circ} \mathrm{C}$ and $450{ }^{\circ} \mathrm{C}$ effectively produces a $(1 \times 1)$ and $(2 \times 1)$ S-passivated $\operatorname{InAs}(100)$ surface. A few ML PTCDA deposition onto the $\operatorname{InAs}(100)-(4 \times 2) / c(8 \times 2)$ substrate form a flat-lying ordered PTCDA-covered substrate. The ringlike diffraction patterns were observed when $\mathrm{CuPc}$ molecules are deposited onto this PTCDA-covered InAs(100) substrate, indicating that the $2 \mathrm{D}$ ordered $\mathrm{CuPc}$ overlayer forms and the overlayer structure is dominantly determined by the intermolecular interactions rather than substrate-molecule interactions. In the case of CuPc deposition on S-passivated InAs(100) surfaces, however, no ordered LEED patterns were observed.

We have calculated the vdW intermolecular interaction energies for both systems in order to rationalise this structural difference. For CuPc unit cells on PTCDA layer, the planar layered $\mathrm{CuPc}$ structure is more stable than the $\alpha$-herringbone $\mathrm{CuPc}$ structure, consistent with the experimental LEED results. By contrast, the $\alpha$-herringbone structure is more stable on a $\mathrm{S}-(1 \times 1)$ layer than the planar layered structure, indicating that the CuPc molecules cannot form flat-lying 2D ordered structure, since the substrate-molecule ( $\mathrm{S}-\mathrm{CuPc}$ ) interactions cannot overcome the penalty of the intermolecular interaction energy arising from the deviation of their native herringbone structure. It is clear from our results that the lattice structure during the initial stage of $\mathrm{CuPc}$ thin film growth in molecular heterolayer structures is influenced strongly by the intermolecular interactions at the interface.

\section{References}

1. Kowalsky, W.; Benstem, T.; Bohler, A.; Dirr, S.; Johannes, H.-H.; Metzdorf, D.; Neuner, H.; Schöbel, J.; Urbach, P. Phys. Chem. Chem. Phys. 1999, 1, 1719.

2. Shalav, A. Prog. Photovolt: Res. Appl. 2009, 17, 513 and references therein.

3. Hook, D. E.; Fritz, T.; Ward, M. D. Adv. Mater. 2001, 13, 227.

4. Koma, A. Prog. Cryst. Growth Charact. 1995, 30, 129.

5. Rochet, F.; Dufour, G.; Roulet, H.; Motta, N.; Sgarlata, A.; Piancastelli, M. N.; Crescenzi, M. De Surf. Sci. 1994, 319, 10.

6. Umbach, E.; Sokolowski, M.; Fink, R. Appl. Phys. A 1996, 63, 565.

7. Sandroff, C. J.; Nottenburg, N.; Bishoff, J. -C.; Baht, R. Appl. Phys. Lett. 1987, 51, 33.

8. Oigawa, H.; Fan, J. -F.; Nannichi, Y.; Ando, K.; Saiki, K.; Koma, A. Jpn. J. Appl. Phys. Part 2 1989, 28, L340.

9. Lowe, M. J.; Veal, T. D.; McConville, C. F.; Bell, G. R.; Tsukamoto, S.; Koguchi, N. Surf. Sci. 2003, 523, 179.

10. Chung, C. -H.; Yi, S. I.; Weinberg, W. H. J. Vac. Sci. Technol. A 1997, 15, 1163.

11. Sugahara, H.; Oshima, M.; Klauser, R.; Oigawa, H.; Nannich, Y. Surf. Sci. 1991, 242, 335.

12. Moriaty, P.; Murphy, B.; Roberts, L.; Cafolla, A. A.; Hughes, G.; Koenders, L.; Bailey, P. Phys. Rev. B 1994, 50, 14237.

13. Wilmsen, C. W.; Geib, K. M.; Shim, J.; Iyer, R.; Lile, D. L.; Pouch, J. J. J. Vac. Sci. Technol. B 1989, 7, 851.

14. McGovern, I. T. Appl. Surf. Sci. 2000, 166, 196.

15. Nannichi, Y.; Fan, J.-F.; Koma, A. Jpn. J. Appl. Phys. 1988, 27, L2367.

16. Lin, Y. J.; Liu, B. Y.; Chin, Y. M. Thin Solid Films 2009, 517, 5508.

17. Heutz, S.; Jones, T. S. J. Appl. Phys. 2002, 92, 3039.

18. Yim, S.; Jones, T. S. Phys. Rev. B 2003, 67, 165308.

19. Bell, G. R.; McConville, C. F.; Jones, T. S. Appl. Surf. Sci. 1996, 104-105, 17.

20. Fukuda, Y.; Suzuki, Y.; Sanada, N.; Shimomura, M.; Masuda, S. Phys. Rev. B 1997, 56, 1084.

21. Kendrick, C.; Kahn, A. J. Cryst. Growth 1997, 181, 181.

22. Ogawa, T.; Kuwamoto, K.; Isoda, S.; Kobayashi, T.; Karl, N. Acta Crystal. B: Struct. Sci. B 1999, 55, 123.

23. Halgren, T. A. J. Am. Chem. Soc. 1992, 114, 7827.

24. Nakamura, M.; Tokumoto, H. Surf. Sci. 1998, 398, 143.

25. Mastryukov, V.; Ruan, C.; Fink, M.; Wang, Z.; Pachter, R. J. Mol. Struct. 2000, 556, 225.

26. Yim, S.; Jones, T. S. Surf. Sci. 2002, 521, 151.

27. Yim, S.; Jones, T. S. J. Phys. Condens. Matt. 2003, 15, S2631.

28. Ashida, M. Bull. Chem. Soc. Jpn. 1966, 39, 2625. 